\title{
Notch signaling molecules as prognostic biomarkers for non-small cell lung cancer
}

\author{
MENG-MENG JIN ${ }^{1}$, YUAN-ZI YE ${ }^{1}$, ZHEN-DONG QIAN $^{2}$ and YAN-BEI ZHANG ${ }^{1}$ \\ ${ }^{1}$ Department of Respiratory Medicine, Anhui Geriatric Institute, The First Affiliated Hospital of Anhui Medical University, \\ Hefei, Anhui 230022; ${ }^{2}$ Department of Respiratory Medicine, Colored Metal General Staff Hospital of Tongling, \\ Tongling, Anhui 244000, P.R. China
}

Received November 2, 2014; Accepted July 28, 2015

DOI: $10.3892 / \mathrm{ol} .2015 .3662$

\begin{abstract}
Notch family proteins have been reported to be associated with the initiation and development of various types of tumors. The present study used a prospective design to investigate the role of Notch proteins as novel biomarkers that are capable of predicting the survival outcome for patients with non-small cell lung cancer (NSCLC). The protein expression of Notch 1, Notch 3 and their ligands, Jagged 1 and Delta-like 4, was examined using immunohistochemistry in NSCLC tissues and adjacent non-cancerous lung tissues from 101 patients who underwent surgical treatment. The expression was also correlated with clinicopathological parameters and overall survival (OS). High Notch 1 protein expression was observed in 55.4\% (56/101) of NSCLC samples and high Notch 3 expression was observed in $53.5 \%$ (54/101). The nuclear expression of Notch 3 was significantly associated with the lymph node status $(\mathrm{P}=0.0026)$ and tumor-node-metastasis $(\mathrm{TNM})$ stage $(\mathrm{P}<0.0001)$, while the coexpression of Notch 1 plus Notch 3 was associated with lymph node status $(\mathrm{P}=0.0056)$, TNM stage $(\mathrm{P}=0.0001)$ and the histological grading $(\mathrm{P}=0.0359)$. In the survival analyses, the high expression of Notch 1 and Notch 3 exhibited an additive effect toward a poorer OS compared with a subtype with low coexpression for the two proteins $(\mathrm{P}<0.001)$, with high nuclear Notch 3 expression in the NSCLC patients maintaining independent prognostic significance for the outcome on multivariate analysis. These data further demonstrate a central role
\end{abstract}

Correspondence to: Professor Yan-Bei Zhang, Department of Respiratory Medicine, Anhui Geriatric Institute, The First Affiliated Hospital of Anhui Medical University, 218 Ji Xi Road, Hefei, Anhui 230022, P.R. China

E-mail: zhangyanbei1963@126.com

Abbreviations: NSCLC, non-small cell lung cancer; JAG1, Jagged 1; DLL4, Delta-like 4; PBS, phosphate-buffered saline; $\mathrm{HR}$, hazard ratio; CI, confidence interval; FVC, forced vital capacity

Key words: Notch 3, Notch 1, immunohistochemistry, non-small cell lung cancer, prognosis for Notch signaling in NSCLC and the significance of Notch 3 as a prognostic indicator of a poorer survival for patients with resected NSCLC.

\section{Introduction}

Lung cancer is the most common type of cancer worldwide $(1,2)$. Non-small cell lung cancer (NSCLC) accounts for $80-85 \%$ of all lung cancers, and although the surgical resection of early-stage tumors confers the greatest potential for long-term survival, 30-60\% of patients with disease stages IB to IIIA succumb within 5 years of surgery $(3,4)$. Therefore, more useful prognostic factors for those patients who have undergone a resection may enable a more accurate prediction of the outcome and could identify those patient groups with poor survival who may benefit from a more precise indication of the efficacy of treatment.

In hematological malignancies, the role for Notch is well established, while more recent studies have demonstrated the significance of Notch activity in the initiation and progression of solid tumors (5-9). The mammalian Notch receptor family consists of four type I transmembrane receptors (known as Notch 1-4), all of which have been implicated in human cancer. There are also five known Notch ligands in mammals, namely Jagged 1 (JAG1), JAG2, Delta-like 1 (DLL1), DLL3 and DLL4, which undergo processing that is similar to Notch processing. The Notch receptor undergoes multiple proteolytic cleavages upon ligand binding. The final cleavage (S3 cleavage) by the $\gamma$-secretase complex results in the release of the active Notch intracellular domain from the plasma membrane and its subsequent translocation into the nucleus (10). It is the S3 cleavage that is targeted by a class of compounds known as the $\gamma$-secretase inhibitors (GSIs). Therefore, treatment with GSIs blocks the terminal cleavage and release from the plasma membrane, preventing Notch signaling.

It has been demonstrated that Notch 1, Notch 3 and their ligands, JAG1 and DLL4, may be involved in malignant transformation. The activation of Notch 1 signaling appears to sustain the motility, migration and invasion of tumor cells in esophagus squamous cell carcinomas, lingual squamous cell carcinoma, endometrial carcinoma and breast cancer (11-15). Almost all cases of T cell acute lymphoblastic leukemia (T-ALL), and colorectal, pancreatic and ovarian 
cancer have been reported to exhibit aberrant Notch 3 expression $(6,8,16,17)$. Our previous study also reported that Notch 3 overexpression was associated with a poor prognosis in NSCLC patients (18). However, the prognostic role of Notch 3 compared with other Notch family members and its association with Notch 1 in human NSCLC remains unclear. In the present study, the expression of Notch 1, Notch 3, JAG1 and DLL4 was investigated in 101 NSCLC tissue samples and association between the expression of the four Notch family members and the clinicopathological variables and prognosis in NSCLC patients was further assessed.

\section{Materials and methods}

Lung cancer specimens. Paraffin-embedded sections were acquired from 101 patients with NSCLC who underwent surgical resection at the Department of Thoracic Surgery, The First Affiliated Hospital of Anhui Medical University (Heifei, Anhui, China) between January 2007 and December 2007. The criteria for study enrollment were as follows: Patients with histopathologically diagnosed NSCLC, no receipt of radiotherapy or chemotherapy prior to surgery, and no history of other tumors. Prior consent and approval was obtained from all NSCLC patients prior to surgery for the use of cancer tissues and adjacent non-cancerous lung tissues for research purposes, and all experiments were conducted adhering to the bioethics rules issued by the Medical Ethics Committee of The First Affiliated Hospital of Anhui Medical University.

The follow-up period ranged from 1 to 60 months, with a median time of 36 months. Informed consent was obtained from all patients for publication of this study. The age of the patients ranged from 32 to 80 years (median, 62 years), and the cohort included 78 males (77.2\%) and 23 females $(22.7 \%)$. The histological diagnosis was determined by hematoxylin and eosin staining according to the new pathological classification of lung cancer (19). Tumor grading and staging were classified according to the new lung cancer staging system developed by the International Association for the Study of Lung Cancer (2009) (20). The results revealed 49 adenocarcinomas (including 9 mucinous adenocarcinomas), 51 squamous cell carcinomas and 1 large cell carcinoma. Furthermore, 18 tumors were well-differentiated, 53 were moderately-differentiated and 30 were poorly-differentiated. Tumor-node-metastasis (TNM) staging revealed that 20 patients were at stage I, 48 were at stage II, 32 were at stage III and 1 was at stage IV.

Ventilatory function and small airway function were detected by the Jaeger Masterscope computer system (Jaeger-Toennies GmbH, Hoechberg, Germany). A predicted forced vital capacity (FVC) of $<80 \%$ or a forced expiratory volume in $1 \mathrm{sec} / \mathrm{FVC}$ of $<70 \%$ was defined as abnormal ventilatory function. A predicted maximal mid-expiratory flow curve $(75 / 25 \%)$ value of $<65 \%$ was defined as abnormal small airway function. Overall survival (OS) was defined as the interval between the date of surgery and the date of mortality. OS was censored at the date of the patient's last tumor assessment, at the date of mortality from other causes or at 5 years post-surgery. Other patient information is summarized in Table I.
Immunohistochemistry. Each tissue was fixed in formalin and embedded in paraffin, then sectioned to a thickness of $3 \mu \mathrm{m}$ and mounted on glass slides. The sections were dewaxed in xylene and dehydrated in graded alcohol, and endogenous peroxidase activity was blocked with $3 \%$ hydrogen peroxide for $10 \mathrm{~min}$. Next, the sections were subjected to antigen retrieval with $10 \mathrm{mmol} / \mathrm{l}$ citrate buffer solution $(\mathrm{pH} 0)$ for $20 \mathrm{~min}$ in a microwave oven at $700 \mathrm{~W}$. Subsequently, the sections were incubated with $10 \%$ goat serum albumin to eliminate non-specific binding and then they were incubated overnight at $4^{\circ} \mathrm{C}$ with the following primary antibodies: Rabbit polyclonal Notch 1 (1:100 dilution; Santa Cruz Biotechnology, Inc., Dallas, TX, USA), rabbit polyclonal Notch 3 (1:50 dilution; Santa Cruz Biotechnology, Inc.,), rabbit polyclonal JAG1 (1:50 dilution; Santa Cruz Biotechnology, Inc.) and rabbit polyclonal Delta-4 (1:100 dilution; Rockland Immunochemicals, Gilbertsville, PA, USA). Sections were then incubated with the appropriate secondary antibodies (goat anti-rabbit immunoglobulin G; 1:50 dilution; ZSGB-BIO, Beijing, China) at room temperature for $60 \mathrm{~min}$. After sufficient phosphate-buffered saline (PBS) rinses, diaminobenzidine was used as chromogen and the sections were counterstained with hematoxylin. The slides were counterstained with hematoxylin, then dehydrated and coverslipped. Samples incubated with PBS instead of primary antibodies were used as negative controls.

Evaluation of immunohistochemical staining. All stained slides were independently evaluated and scored by three pathologists who had no knowledge of the patients' clinical information. If a disagreement occurred, the slides were re-examined to obtain a final consensus. Positive staining was evaluated in at least five areas at $\mathrm{x} 400$ magnification. The mean percentage of positive cells were scored as follows: $0,0 \% ; 1,1-25 \%$; 2, 26-50\%; 3, 51-75\%; and 4, 76-100\%. The staining intensity was scored as follows: 0 , negative; 1 , weak; 2 , moderate; and 3 , strong. A final histological score was obtained for each case by multiplying the percentage staining and intensity scores. Protein expression levels were further analyzed by classifying histological scores as low expression (histological score $<5$ ) and as high expression (histological score $\geq 5$ ).

Statisticalanalysis. All statistical analyses were performed with R software for Windows (version 2.15.3; http://cran.r-project. org/web/packages/dlnm/vignettes/dlnmOverview.pdf). The associations between various clinicopathological parameters and the expression of Notch 1, Notch 3, JAG1 or DLL4 was evaluated using $\chi^{2}$ or Fisher's exact tests. Kaplan-Meier and log-rank methods were used to draw and evaluate the significance of survival curves. Multivariate Cox proportional hazards regression analysis was used to identify independent prognostic factors for survival rates following univariate survival analysis. $\mathrm{P}<0.05$ was used to indicate a statistically significant difference.

\section{Results}

Notch 1 expression and the association with clinicopathological parameters. Notch 1 immunoreactivity is shown in Fig. 1. Notch 1 protein expression was localized mainly in the cytoplasm, with certain tumor cells showing membranous 


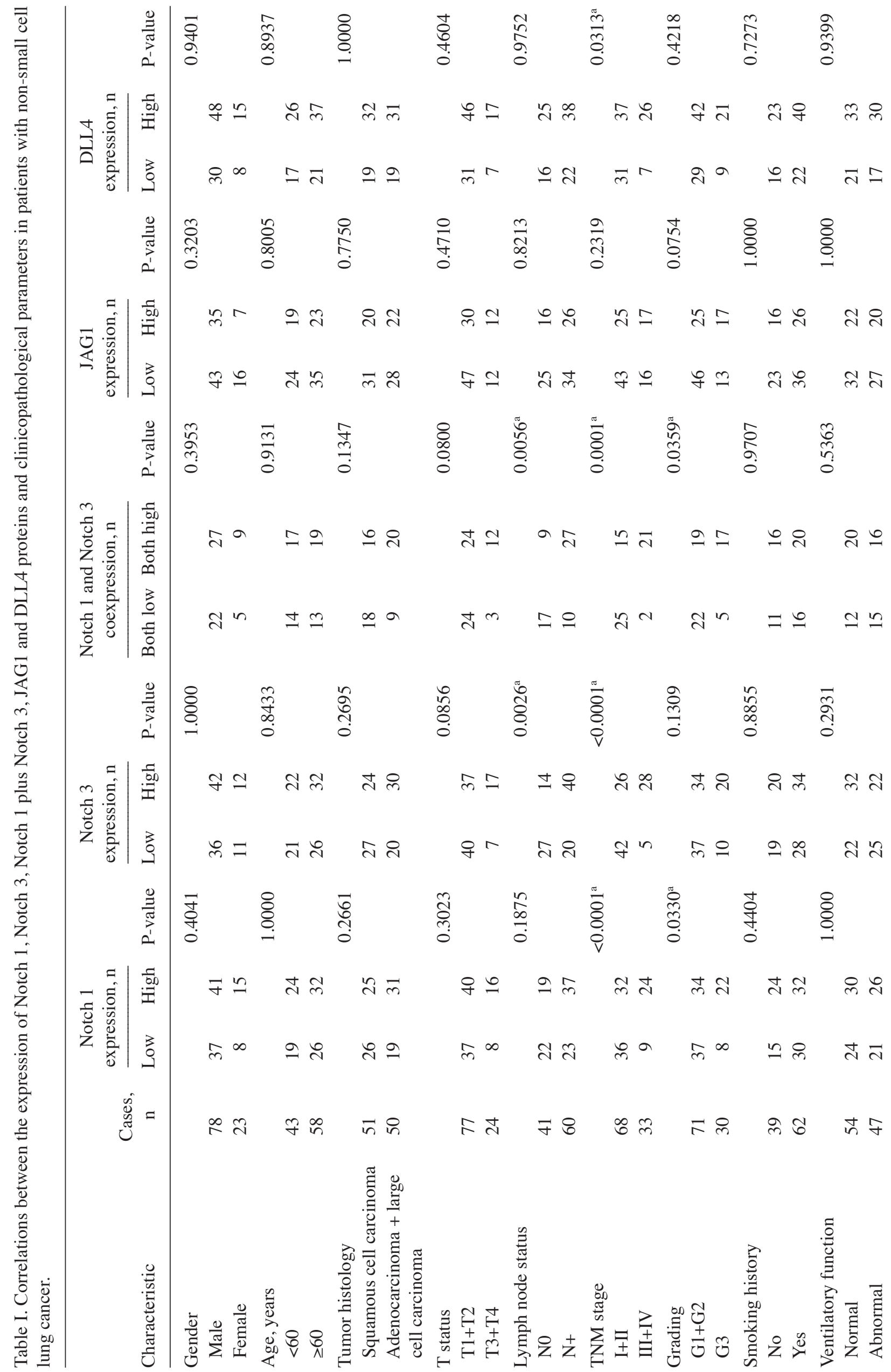


expression. The staining was absent in the adjacent non-cancerous normal lung tissues (Fig. 1A). High Notch 1 protein expression was observed in 55.4\% (56/101) of the NSCLC samples (Fig. 1C) and low expression levels were detected in $44.6 \%$ (45/101) of the tumor sections (Fig. 1B). As summarized in Table I, the high expression of Notch 1 was significantly correlated with TNM stage $(\mathrm{I}+\mathrm{II}$ vs. III+IV) $(\mathrm{P}=0.0264)$ and histological grading $(\mathrm{G} 1+\mathrm{G} 2$ vs. $\mathrm{G} 3)(\mathrm{P}=0.0330)$ in the NSCLC tissues.

Notch 3 expression and the association with clinicopathological parameters. Notch 3 immunoreactivity is shown in Fig. 2. Notch 3 was predominantly localized in the nucleus of the tumor cells. The staining was absent in the adjacent non-cancerous normal lung tissues (Fig. 2A). High Notch 3 protein expression was observed in $53.5 \%$ (54/101) of the NSCLC samples (Fig. 2C) and low expression levels were detected in $46.5 \%$ (47/101) (Fig. 2B). As summarized in Table I, the high expression of Notch 3 was significantly correlated with lymph node status (N0 vs. $\mathrm{N}+)(\mathrm{P}=0.0026)$ and TNM stage (I+II vs. III+IV) $(\mathrm{P}<0.0001)$ in the NSCLC samples.

Association between Notch 1 plus Notch 3 coexpression and clinicopathological characteristics. High coexpression of Notch 1 and Notch 3 was detected in 36 (35.6\%) samples, and low expression was observed in 27 samples (26.7\%). Table I shows that the high coexpression of Notch 1 plus Notch 3 was significantly correlated with lymph node status (N0 vs. N+) $(\mathrm{P}=0.0056)$, TNM stage (I+II vs. III+IV) $(\mathrm{P}=0.0001)$ and histological grading $(\mathrm{G} 1+\mathrm{G} 2$ vs. $\mathrm{G} 3)(\mathrm{P}=0.0359)$.

JAG1 and DLL4 expression and the association with clinicopathological findings. JAG1 and DLL4 immunoreactivity is shown in Fig. 3. JAG1 and DLL4 protein expression was localized mainly in the cytoplasm, with membranous staining. High JAG1 protein expression was observed in $41.6 \%(42 / 101)$ (Fig. 3B) and low expression levels were detected in $58.4 \%$ (59/101) (Fig. 3A). High DLL4 protein expression was observed in $62.4 \%$ (63/101) (Fig. 3D) and low expression levels were detected in 37.6\% (38/101) (Fig. 3C). Table I shows no association between high JAG1 expression and the clinicopathological features in the NSCLC patients ( $\mathrm{P}>0.05)$, while the high expression of DLL4 was significantly correlated with TNM stage (I+II vs. III+IV) $(\mathrm{P}=0.0313)$.

Survival analysis. Follow-up was discontinued for all patients in December 2012. The median follow-up time was 36 months (range, 1-60 months). The overall 5-year survival rate was 45\%, with a median survival time of 56 months. The data indicated that there was a significant difference in OS between the patients with high and low expression of all four Notch family members $(\mathrm{P}<0.05)$, which indicated that the high expression of Notch 1, Notch 3, JAG1 or DLL4 was correlated with a shorter survival time. Furthermore, the subtype with high coexpression of Notch 1 and Notch 3 exhibited a worse outcome than other subtypes (log-rank, 21.227; P<0.001) (Fig. 4).

Results of the univariate analysis with regard to OS for the clinicopathological prognostic factors are shown in Table II. The high expression of the four Notch proteins was found to be a significant indicator of a poor OS $(\mathrm{P}<0.05)$ (Table II). With regard to other parameters, TNM stage, lymph node status and 
Table II. Univariate and multivariate Cox proportional hazards analyses for prognostic factors in patients with non-small cell lung cancer.

\begin{tabular}{|c|c|c|c|}
\hline \multirow[b]{2}{*}{ Variable } & \multicolumn{3}{|c|}{ Overall survival } \\
\hline & HR & $95 \% \mathrm{CI}$ & P-value \\
\hline \multicolumn{4}{|l|}{ Univariate analysis } \\
\hline Gender (female vs. male) & 0.8090 & $0.4026-1.6260$ & 0.5508 \\
\hline Age ( $\geq 60$ vs. $<60$ years $)$ & 0.7849 & $0.4453-1.3830$ & 0.4011 \\
\hline Tumor histology (adenocarcinomas vs. quamous cell carcinoma) & 1.0610 & $0.6021-1.8690$ & 0.8383 \\
\hline T status $(\mathrm{T} 3+\mathrm{T} 4$ vs. $\mathrm{T} 1+\mathrm{T} 2)$ & 1.7960 & $0.9609-3.3570$ & 0.0627 \\
\hline Lymph node status (N+ vs. N0) & 3.5700 & $1.8140-7.0260$ & $<0.0001^{\mathrm{a}}$ \\
\hline TNM stage (III+IV vs. I+II) & 6.4060 & $3.5500-11.560$ & $<0.0001^{\mathrm{a}}$ \\
\hline Grading (G3 vs. G1+G2) & 1.9020 & $1.0480-3.4490$ & $0.0315^{\mathrm{a}}$ \\
\hline Smoking history (yes vs. no) & 1.3560 & $0.7435-2.4730$ & 0.3187 \\
\hline Ventilatory function (abnormal vs. normal) & 1.2590 & $0.7149-2.2190$ & 0.4237 \\
\hline Small airway function (abnormal vs. normal) & 1.7240 & $0.8064-3.6840$ & 0.1550 \\
\hline Anatomical classification (central vs. peripheral) & 1.5630 & $0.8829-2.7680$ & 0.1222 \\
\hline Notch 1 expression (high vs. low) & 2.0800 & $1.1370-3.8040$ & $0.0151^{\mathrm{a}}$ \\
\hline Notch 3 expression (high vs. low) & 3.7220 & $1.9590-7.0720$ & $<0.0001^{\mathrm{a}}$ \\
\hline JAG1 expression (high vs. low) & 1.9760 & $1.1180-3.4940$ & $0.0170^{\mathrm{a}}$ \\
\hline DLL4 expression (high vs. low) & 1.9620 & $1.0370-3.7130$ & $0.0348^{\mathrm{a}}$ \\
\hline \multicolumn{4}{|l|}{ Multivariate analysis } \\
\hline Gender (female vs. male) & 0.8756 & $0.3004-2.5523$ & 0.8076 \\
\hline Age ( $\geq 60$ vs. $<60$ years $)$ & 1.0026 & $0.5048-1.9913$ & 0.9940 \\
\hline Tumor histology (adenocarcinomas vs. quamous cell carcinoma) & 1.0097 & $0.5309-1.9202$ & 0.9766 \\
\hline $\mathrm{T}$ status $(\mathrm{T} 3+\mathrm{T} 4 \mathrm{vs} . \mathrm{T} 1+\mathrm{T} 2)$ & 0.8430 & $0.3717-1.9121$ & 0.6827 \\
\hline Lymph node status (N+ vs. N0) & 1.7543 & $0.7431-4.1411$ & 0.1997 \\
\hline TNM stage (III+IV vs. I+II) & 5.1447 & $2.1070-12.5621$ & $0.0003^{\mathrm{a}}$ \\
\hline Grading (G3 vs. G1+G2) & 1.5180 & $0.7615-3.0263$ & 0.2357 \\
\hline Smoking history (yes vs. no) & 0.8977 & $0.3527-2.2850$ & 0.8209 \\
\hline Ventilatory function (abnormal vs. normal) & 1.4652 & $0.6636-3.2350$ & 0.3445 \\
\hline Small airway function (abnormal vs. normal) & 1.7839 & $0.6867-4.6345$ & 0.2347 \\
\hline Anatomical classification (central vs. peripheral) & 1.8915 & $0.9343-3.8291$ & 0.0765 \\
\hline Notch 1 expression (high vs. low) & 0.9105 & $0.3855-2.1510$ & 0.8308 \\
\hline Notch 3 expression (high vs. low) & 2.5126 & $1.1383-5.5460$ & $0.0226^{\mathrm{a}}$ \\
\hline JAG1 expression (high vs. low) & 1.1646 & $0.4850-2.7970$ & 0.7331 \\
\hline DLL4 expression (high vs. low) & 1.2515 & $0.5477-2.8600$ & 0.5947 \\
\hline
\end{tabular}

${ }^{\mathrm{a}} \mathrm{P}<0.05$. JAG1, Jagged 1; DLL, Delta-like 4; CI, confidence interval; HR, hazard ratio; TNM, tumor-node-metastasis.
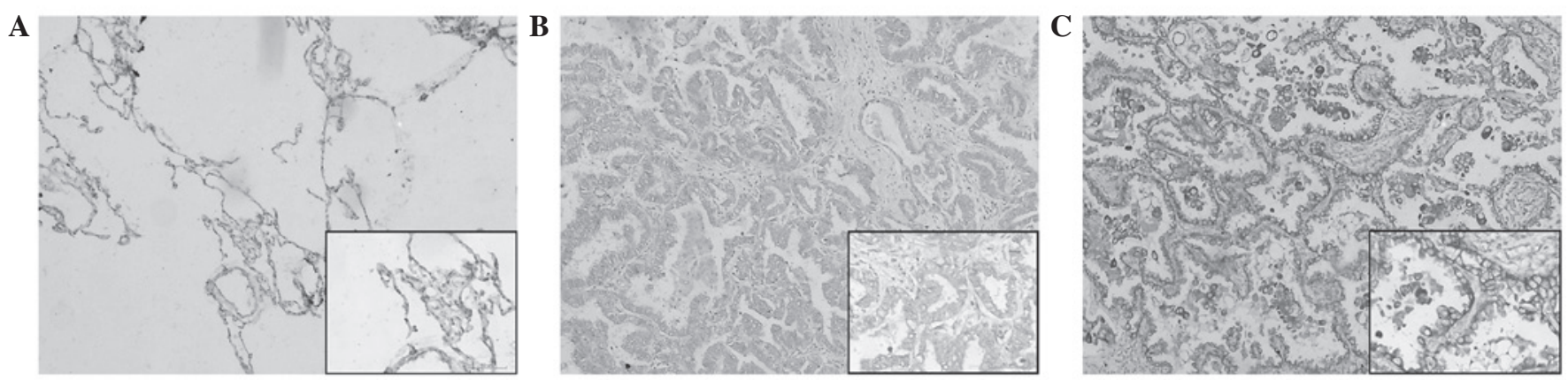

Figure 1. Representative immunohistochemical staining for Notch 1 protein in primary non-small cell lung cancer (NSCLC) tissues and adjacent non-cancerous lung tissues. (A) Negative expression of Notch 1 protein in adjacent non-cancerous lung tissues. (B) Low expression of Notch 1 in primary NSCLC specimens. (C) High expression of Notch 1 protein in primary NSCLC specimens. Magnification, x100; inset images, x400. 
A

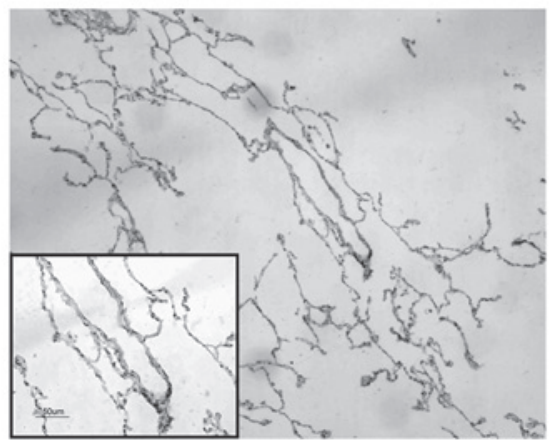

$\mathbf{B}$

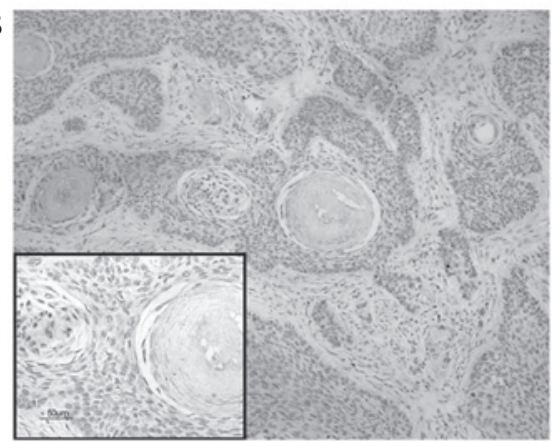

$\mathbf{C}$

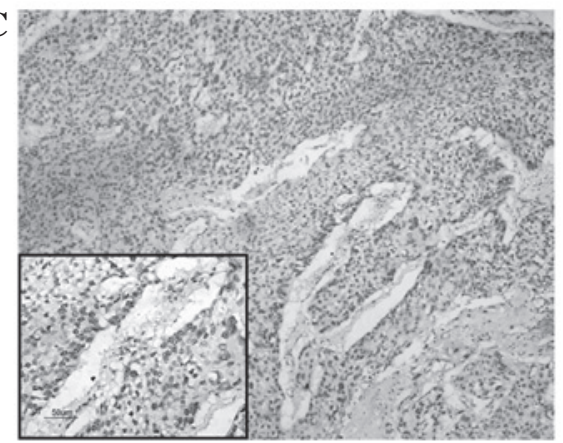

Figure 2. Representative immunohistochemical staining for Notch 3 protein in primary non-small cell lung cancer (NSCLC) tissues and adjacent non-cancerous lung tissues. (A) Negative expression of Notch 3 protein in adjacent non-cancerous lung tissues. (B) Low expression of Notch 3 in primary NSCLC specimens. (C) High expression of Notch 3 protein in primary NSCLC specimens. Magnification, x100; inset images, x400.
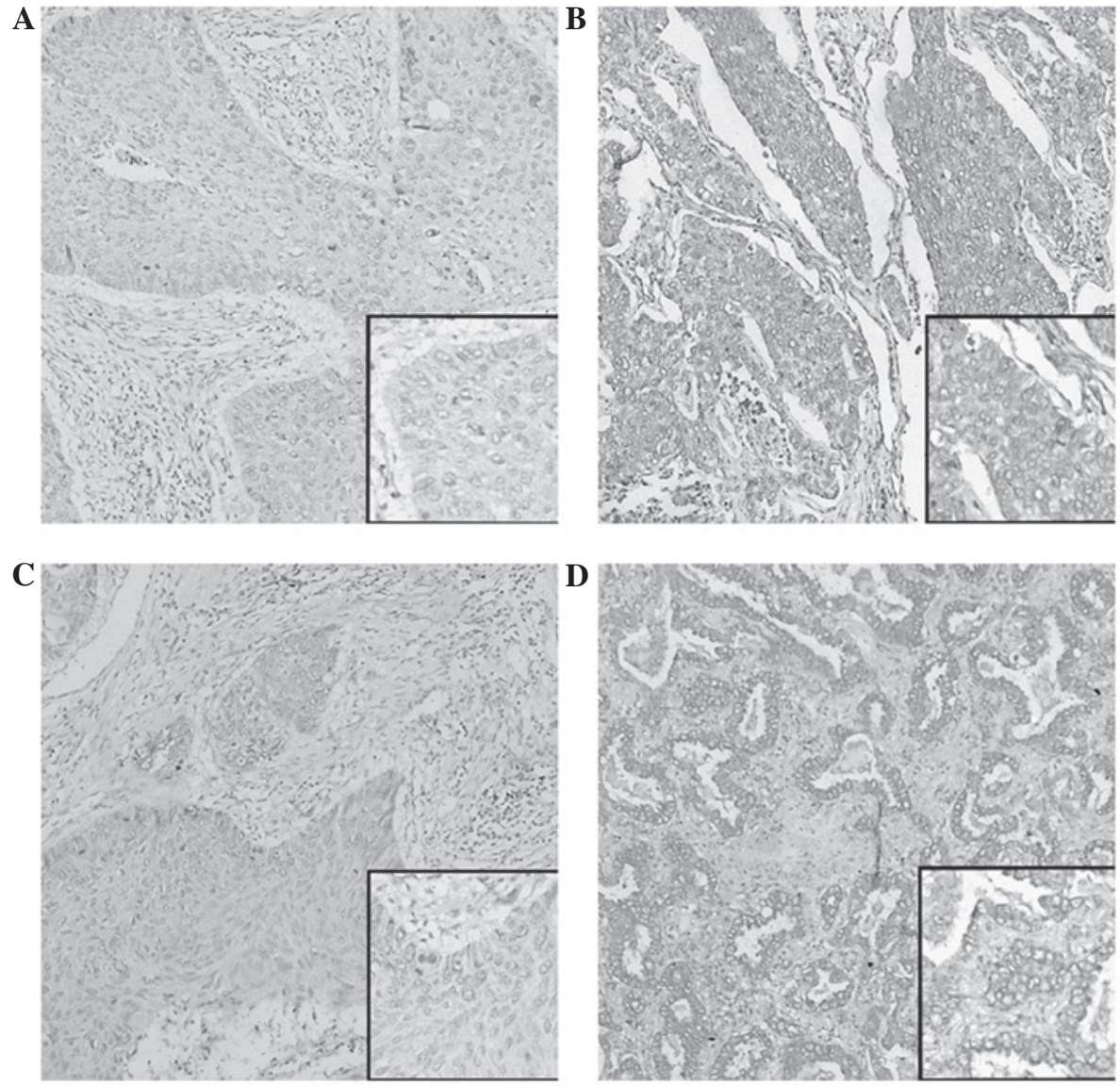

Figure 3. Representative immunohistochemical staining for Jagged 1 (JAG1) and Delta-like 4 (DLL4) protein in primary non-small cell lung cancer (NSCLC) tissues and adjacent non-cancerous lung tissues. (A) Low expression of JAG1 in primary NSCLC specimens. (B) High expression of JAG1 protein in primary NSCLC specimens. (C) Low expression of DLL4 in primary NSCLC specimens. (D) High expression of DLL4 protein in primary NSCLC specimens. Magnification, $\mathrm{x} 100$; inset images, $\mathrm{x} 400$.

histological grading were determined as positive significant prognostic factors for OS $(\mathrm{P}<0.05)$ (Table II). In the multivariate analysis, Notch 3 expression was found to be an independent prognostic factor of OS in patients with NSCLC compared with the other three Notch family members ( $\mathrm{P}=0.0226)$ (Table II).

\section{Discussion}

In this study, Notch 1, Notch 3, JAG1 and DLL4 protein expression was examined immunohistochemically in a well-defined cohort of NSCLC patients and the expression levels were correlated to clinical parameters and patient outcome.

The phenotypic outcome of Notch signaling is often context-dependent. Different Notch receptors play different, even opposing, roles in tumor development, showing the complexity of Notch signaling in cancer. The expression of Notch 3 has been found to be significantly decreased in human tumor cell lines, and in primary human breast cancer and melanoma samples compared with normal control tissues (21). However, the present data demonstrated that Notch 3 is 

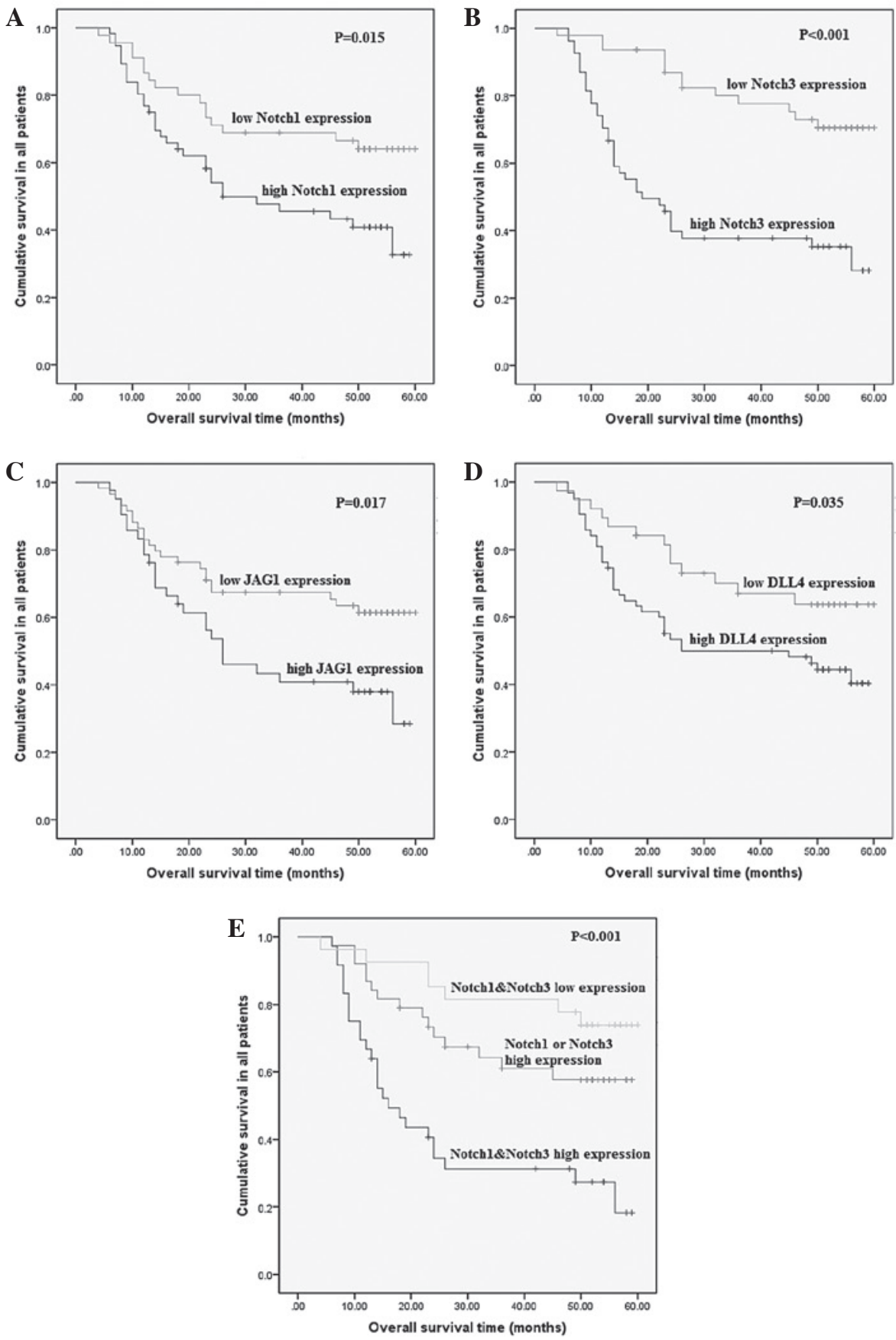

Figure 4. Kaplan-Meier curves of survival patients with (A) Notch 1, (B) Notch 3, (C) Jagged 1 (JAG1), (D) Delta-like 4 (DLL4), and (E) Notch 1 plus Notch 3 expression. The log-rank test was used to calculate P-values.

ubiquitously expressed in NSCLC. The majority of tumors showed high levels of nuclear Notch 3 expression. This expression pattern closely resembles data previously recorded in pancreatic ductal adenocarcinomas (22), while certain more recent studies have demonstrated that the immunoreactivity of Notch 3 is observed mainly in the cytoplasm of tumor cells with or without nuclei staining $(8,9,13)$. The mechanism of this phenomenon and how Notch 3 exerts its function in NSCLC remain unclear and require further research. Most notably, in the present study, the upregulated expression of Notch 3 was a predictor of different aggressive tumor behaviors, such as advanced clinical stage and lymph node metastasis. In lung cancer, Notch 1 is known to suppress tumor proliferation under normoxia, but in hypoxia, it exhibits a converse role in tumor promotion (23). In the present study, Notch 1 expression, with levels varying from low to high, was demonstrated in a number of NSCLC patients. Notch 1 expression was localized in the cytoplasm, with membranous expression, similar to the results found in studies of other tissue tumors (11-14). Furthermore, Notch 1 expression was found to be associated with TNM stage and histological grading, which also indicated the impact of Notch 1 expression on the progression of NSCLC. The results strongly suggested that Notch 1 and Notch 3 may play key roles in the advancement of NSCLC.

As Notch receptor ligands, JAG1 and DLL4 have been found to function in cancer progression and 
metastasis (24-28). In the present study, the expression of the JAG1 and DLL4 notch ligands was examined in NSCLC tissues and the expression levels were compared with clinical parameters. However, no correlation was found between the JAG1 expression levels and clinical parameters in NSCLC. In addition, it was shown that in tumor tissues, high levels of DLL4 expression were correlated with TNM stage, which suggested that DLL4 may be associated with the progression of NSCLC.

Specific foci, including comborbidities, smoking history, and general clinical and demographic features have been investigated in previous analyses of prognostic factors in surgically resected NSCLC. Pathological TNM stage, age and gender were all determined to be independent prognostic factors for survival, with pathological TNM stage representing the most important prognostic factor (29). However, the outcome may vary depending on several biochemical and clinical parameters, even among those patients with clinically localized disease. In the present study, Kaplan-Meier analysis of the survival curves showed a significantly worse overall survival rate for patients with tumors that exhibited high Notch 1 , Notch 3 , JAG1 or DLL4 protein levels, indicating that high Notch 1, Notch 3, JAG1 and DLL4 protein levels are markers of a poor prognosis for patients with NSCLC. Furthermore, the present results showed that the high coexpression of Notch 1 and Notch 3 predicted a worse outcome compared with the Notch 1 or Notch 3 high expression subtypes. These data suggested that the coexpression of Notch 1 and Notch 3 has additive roles in the biological behavior of NSCLC. Moreover, univariate analysis showed that high Notch 1, Notch 3, JAG1 and DLL4 expression, TNM stage and tumor histological grading were risk factors for a poor prognosis in NSCLC patients, but multivariate analysis showed that a high level of Notch 3 expression was the only independent risk factor of prognosis for NSCLC patients, suggesting that the level of Notch 3 expression in NSCLC tissue samples may be used as a more useful prognostic marker compared with Notch 1 in NSCLC patients. Previous studies have demonstrated that cellular proliferation is significantly reduced by $\gamma$-secretase inhibitor $(30,31)$ and that the apoptosis of Notch 3 -expressing cells is induced. In lung cancer, the inhibition of Notch activation using a $\gamma$-secretase inhibitor is a potential novel approach for targeted therapy (32). Thus, in NSCLC patients, Notch 3 expression may represent a useful additive prognostic marker to the TNM staging system and thus, such patients are good candidates for receiving aggressive adjuvant targeted therapy.

In summary, the present findings demonstrated that high levels of Notch 1 and Notch 3 expression were significantly correlated with NSCLC progression and a poor prognosis. Furthermore, Notch 3 expression can be used as an adjunct to the TNM staging system to improve prognostication for individual patients. Additionally, it can be concluded that Notch 3 may present as a more attractive prognostic biomarker associated with NSCLC compared with Notch 1. Therefore, we hypothesize that targeting Notch 3 in specific cell types may be more useful than targeting Notch 1 . In the near future, targeting the Notch 3 pathway may be used for the formation of novel preventive and therapeutic strategies for NSCLC.

\section{Acknowledgements}

This study was funded by grants from the Key Programs of the Educational Commission of Anhui Province (no. KJ2011A178), the Natural Science Foundation of Science and Technology Department of Anhui Province (no. 1208085MH146), and the Scientific and Technological Programs of Science and Technology Department of Anhui Province (no. 1501041144).

\section{References}

1. Jemal A, Bray F, Center MM, Ferlay J, Ward E and Forman D: Global cancer statistics. CA Cancer J Clin 61: 69-90, 2011.

2. Bray F, Jemal A, Grey N, Ferlay J and Forman D: Global cancer transitions according to the Human Development Index (2008-2030): A population-based study. Lancet Oncol 13: 790-801, 2012.

3. Sangha R, Price J and Butts CA: Adjuvant therapy in non-small cell lung cancer: Current and future directions. Oncologist 15: 862-872, 2010

4. Raso MG and Wistuba II: Molecular pathogenesis of early-stage non-small cell lung cancer and a proposal for tissue banking to facilitate identification of new biomarkers. J Thorac Oncol 2 (7 Suppl 3): S128-S135, 2007.

5. Ranganathan P, Weaver KL and Capobianco AJ: Notch signalling in solid tumours: A little bit of everything but not all the time. Nat Rev Cancer 11: 338-351, 2011.

6. Rahman MT, Nakayama K, Rahman M, Katagiri H, Katagiri A, Ishibashi T, Ishikawa M, Iida K, Nakayama S, Otsuki Y and Miyazaki K: Notch 3 overexpression as potential therapeutic target in advanced stage chemoresistant ovarian cancer. Am J Clin Pathol 138: 535-544, 2012.

7. Jonusiene V, Sasnauskiene A, Lachej N, Kanopiene D, Dabkeviciene D, Sasnauskiene S, Kazbariene B and Didziapetriene J: Down-regulated expression of Notch signaling molecules in human endometrial cancer. Med Oncol 30: 438, 2013.

8. Mann CD, Bastianpillai C, Neal CP, Masood MM, Jones DJ, Teichert F, Singh R, Karpova E, Berry DP and Manson MM: Notch 3 and HEY-1 as prognostic biomarkers in pancreatic adenocarcinoma. PLoS One 7: e51119, 2012.

9. Kang H, An HJ, Song JY, Kim TH, Heo JH, Ahn DH and Kim G: Notch 3 and Jagged 2 contribute to gastric cancer development and to glandular differentiation associated with MUC2 and MUC5AC expression. Histopathology 61: 576-586, 2012.

10. Kopan R and Ilagan MX: The canonical Notch signaling pathway: Unfolding the activation mechanism. Cell 137: 216-233, 2009.

11. Liu J, Fan H, Ma Y, Liang D, Huang R, Wang J, Zhou F, Kan Q, Ming L, Li H, et al: Notch 1 is a 5-fluorouracil resistant and poor survival marker in human esophagus squamous cell carcinomas. PLoS One 8: e56141, 2013.

12. Yu B, Wei J, Qian X, Lei D, Ma Q and Liu Y: Notch 1 signaling pathway participates in cancer invasion by regulating MMPs in lingual squamous cell carcinoma. Oncol Rep 27: 547-552, 2012.

13. Mitsuhashi Y, Horiuchi A, Miyamoto T, Kashima H, Suzuki A and Shiozawa T: Prognostic significance of Notch signalling molecules and their involvement in the invasiveness of endometrial carcinoma cells. Histopathology 60: 826-837, 2012.

14. Reedijk M, Odorcic S, Chang L, et al: High-level coexpression of JAG1 and Notch 1 is observed in human breast cancer and is associated with poor overall survival. Cancer Res 65: 8530-8537, 2005.

15. Wang J, Fu L, Gu F and Ma YJ: Notch 1 is involved in migration and invasion of human breast cancer cells. Oncol Rep 26: 1295-1303, 2011.

16. Bellavia D, Campese AF, Checquolo S, et al: Combined expression of pTalpha and Notch 3 in T cell leukemia identifies the requirement of preTCR for leukemogenesis. Proc Natl Acad Sci U S A 99: 3788-3793, 2002.

17. Serafin V, Persano L, Moserle L, Esposito G, Ghisi M, Curtarello M, Bonanno L, Masiero M, Ribatti D, Stürzl M, et al: Notch 3 signalling promotes tumour growth in colorectal cancer. J Pathol 224: 448-60, 2011.

18. Ye YZ, Zhang ZH, Fan XY, Xu XL, Chen ML, Chang BW and Zhang YB: Notch 3 overexpression associates with poor prognosis in human non-small-cell lung cancer. Med Oncol 30: $595,2013$. 
19. Travis WD, Brambilla E and Riely GJ: New pathologic classification of lung cancer: Relevance for clinical practice and clinical trials. J Clin Oncol 31: 992-1001, 2013.

20. Detterbeck FC, Boffa DJ and Tanoue LT: The new lung cancer staging system. Chest 136: 260-271, 2009.

21. Cui H, Kong Y, Xu M and Zhang H: Notch 3 functions as a tumor suppressor by controlling cellular senescence. Cancer Res 73: 3451-3459, 2013.

22. Doucas H, Mann CD, Sutton CD, et al: Expression of nuclear Notch 3 in pancreatic adenocarcinomas is associated with adverse clinical features and correlates with the expression of STAT3 and phosphorylated Akt. J Surg Oncol 97: 63-68, 2008.

23. Chen Y, De Marco MA, Graziani I, et al: Oxygen concentration determines the biological effects of NOTCH-1 signaling in adenocarcinoma of the lung. Cancer Res 67: 7954-7959, 2007.

24. Simon DP, Giordano TJ and Hammer GD: Upregulated JAG1 enhances cell proliferation in adrenocortical carcinoma. Clin Cancer Res 18: 2452-2464, 2012.

25. Steg AD, Katre AA, Goodman B, Han HD, Nick AM, Stone RL, Coleman RL, Alvarez RD, Lopez-Berestein G, Sood AK and Landen CN: Targeting the notch ligand JAGGED1 in both tumor cells and stroma in ovarian cancer. Clin Cancer Res 17: 5674-5685, 2011.

26. Koukourakis MI, Giatromanolaki A, Sivridis E, Gatter KC and Harris AL: High DLL4 expression in tumour-associated vessels predicts for favorable radiotherapy outcome in locally advanced squamous cell head-neck cancer (HNSCC). Angiogenesis 16: 343-351, 2013.
27. Liu Z, Fan F, Wang A, Zheng S and Lu Y: Dll4-Notch signaling in regulation of tumor angiogenesis. J Cancer Res Clin Oncol 140: 525-536, 2014.

28. Ishigami S, Arigami T, Uenosono Y, Okumura H, Kurahara H, Uchikado Y, Setoyama T, Kita Y, Kijima Y, Nishizono Y, et al: Clinical implications of DLL4 expression in gastric cancer. J Exp Clin Cancer Res 32: 46, 2013.

29. Chansky K, Sculier JP, Crowley JJ, Giroux D, Van Meerbeeck J and Goldstraw P; International staging committee and participating institutions: The international association for the study of lung cancer staging project: Prognostic factors and pathologic TNM stage in surgically managed non-small cell lung cancer. J Thorac Oncol 4: 792-801, 2009.

30. Danza G, Di Serio C, Ambrosio MR, Sturli N, Lonetto G, Rosati F, Rocca BJ, Ventimiglia G, del Vecchio MT, Prudovsky I, et al: Notch 3 is activated by chronic hypoxia and contributes to the progression of human prostate cancer. Int J Cancer 133: 2577-2586, 2013.

31. Chen X, Thiaville MM, Chen L, Stoeck A, Xuan J, Gao M and Shih IeM and Wan TL: Defining Notch 3 target genes in ovarian cancer. Cancer Res 72: 2294-2303, 2012.

32. Konishi J, Kawaguchi KS, Vo H, Haruki N, Gonzalez A, Carbone DP and Dang TP: Gamma-secretase inhibitor prevents Notch 3 activation and reduces proliferation in human lung cancers. Cancer Res 67: 8051-8057, 2007. 